\title{
Complex Integration of Aerodynamic Micro-Foam Generators into Specialized Technological Devices with Artificial Intelligence and Artificial Neural Networks for System Control
}

\author{
Victor Popov \\ Master of Engineering in Computer Science, Cornell University, New York, \\ United States of America \\ Bachelor's Degree in Mechatronics and Robotics, Bauman Moscow State \\ Technical University, Moscow, Russian Federation
}

\begin{abstract}
The system of aerodynamic foam generation is covered in this article as well as its structure and main technological and structural characteristics. The author describes technological and industrial processes related to production of thin filmed micro assemblies from which logically follows the expediency of using micro foam for solving specified objectives, its main advantages as well as reasoning behind choosing the aerodynamic principle for foam generation. Besides the principles of system operation, the author also considered different options for its application in industrial settings. Special attention is focused on the application at lines of photolithographic masking and galvanic coating on the boards of thin filmed micro assemblies, but the author also considers a case for usage as a fuel mixture which leads to reduction of fuel consumption and simplification of the construction of combustion chamber sealing or cylinders of the diesel engine. Author considers in detail the main structural components of the construction of the device for aerodynamic micro foam generation as well as the properties and characteristics of the obtained micro foal primarily due to aerodynamic effect. Thorough description is given to the principle diagram and principles of assembly operation for using the aerodynamic foam generator for various industrial technological processes. Comparative analysis is conducted for the suggested technical object and known technical objects that were discovered during patent search. As a result, the list of properties for significant novelty is elicited and outlined. The described system allows usage of artificial intelligence and machine learning for system control. Analyses of the suggested technology was performed in accordance with the methods and criteria of Theory of Inventive Problem Solving and Algorithms of Inventive Problem Solving.
\end{abstract}

Keywords: Activated foam, aerodynamic foam generator, thin-filmed micro-assembly line, activated fuel mixture, artificial intelligence in control systems

\section{Introduction}

The local technical system (sub-system) for aerodynamic foam generation is new. Thus, for beginning of analytic estimation of its properties and significant differences the author in general terms suggests the following preliminary formulation, that characterizing structural and technological dependencies and connections between main underlying elements of the specified system. A device for foam generation in isolated and flowing in the pipeline liquid stream mainly of organic nature. It includes successively located (in the stream direction) connected to the generator of specified current volume hydrodynamic amplifier of turbulence level and 
connected to the source of compressed gas aerodynamic stimulator of tridimensional microturbulence of the specified current which connects to the conical reflector.

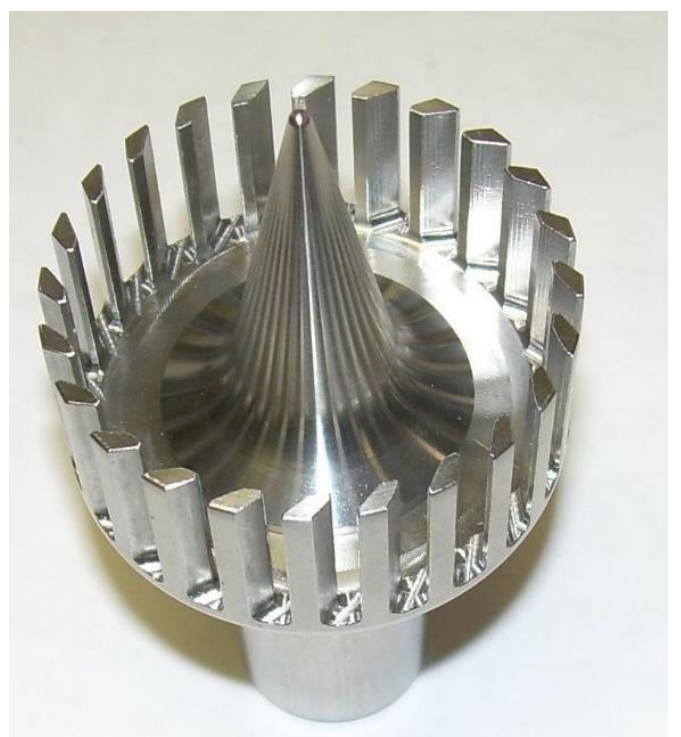

Figure 1. Aerodynamic foam generator made of stainless steel with specially profiled plate that are used for smooth increase in turbulence level in the stream of foamed liquid flowing from the gap-channel between membranes that is combined with conical reflector.

Problem Formulation and Analysis

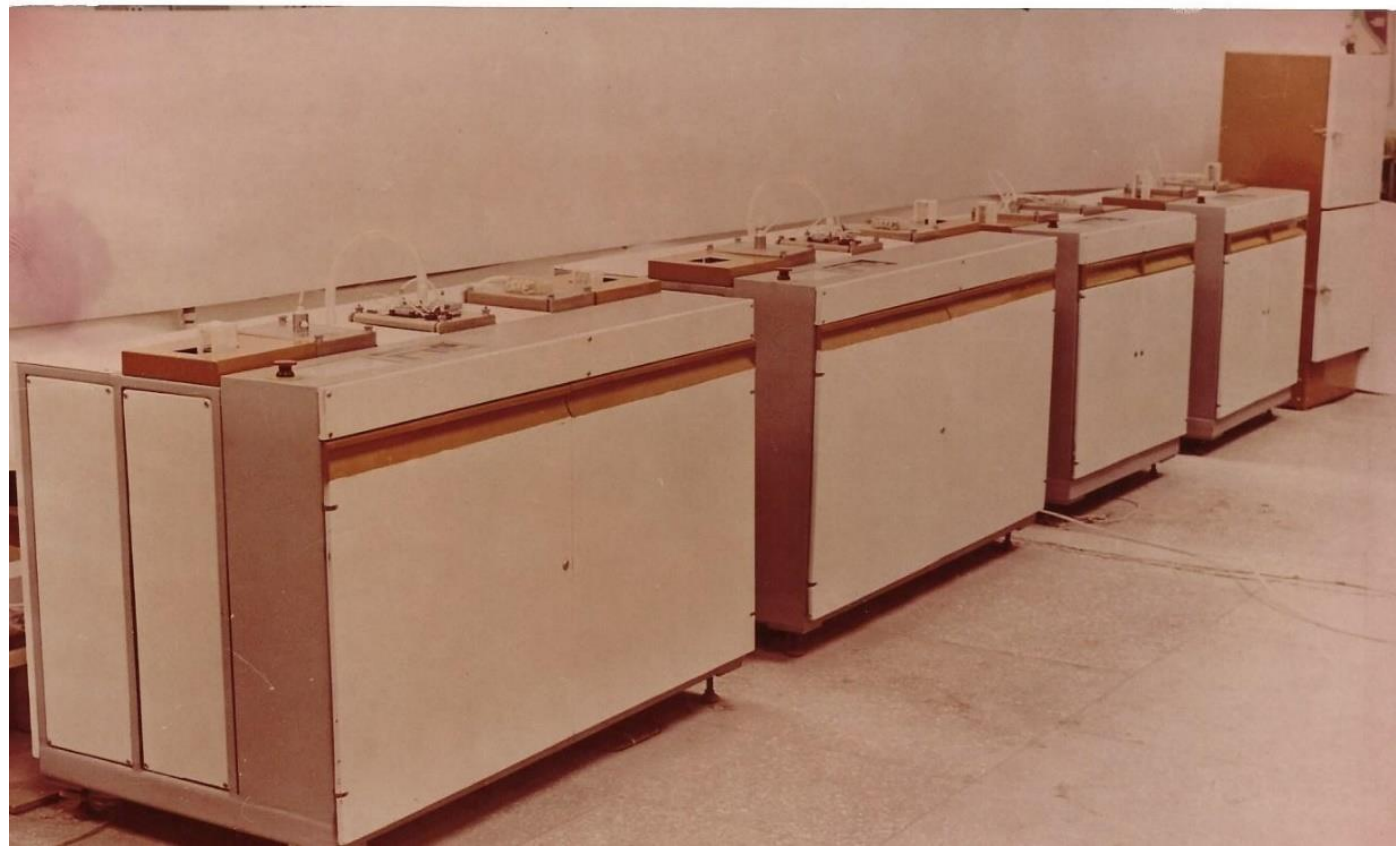

Figure 1.1. Automatic line of galvanic coating on the boards of thin-film micro assembly 
All line plants contain at least two working position (including at least one centrifuge) in combination with two loading-unloading modules and three intermediate modules. Usually every plant has at least one module that is used for preparation of the surface or for cleaning and the time required for this operation should be equal to processing time on other working positions. Thus, the time of efficient work should be minimal and furthermore, the quality of this operation should be in accordance with standard requirements and main criteria of the technology.

When choosing an option for these operations the most expediently is to use aerodynamic foam generators, moreover for both cleaning and regeneration of the technological water, because aerodynamic foam generators are the most efficient technical solutions for this kind of problems.

It is particularly important, that the time required for foam formation is minimal and due to properties and characteristics of foam generator the gas or air bubbles in the resulted foam are totally identical (in terms of size) and the bubble diameter is determined by the distance between base of the reflector and the top plane of generator body. This prevents errors in setup and in the range of pressures and compressed gas consumptions feeding to the foam generator and provides absolutely identical foam parameters and ensures equal conditions for technological operations and transitions of automatic line machines. 


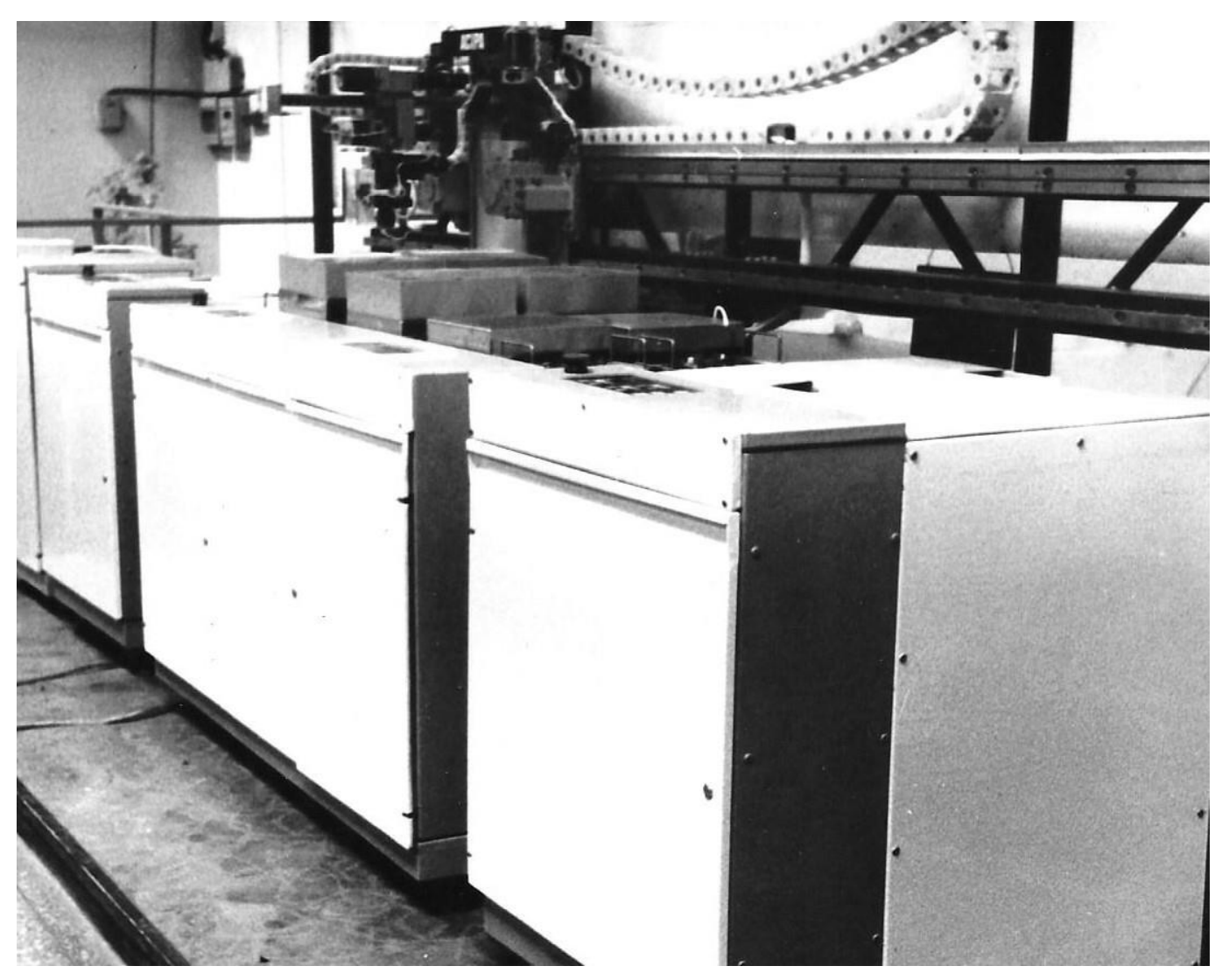

Figure 1 - 2. Adjustable automatic production module for photo-lithography on the boards of thin-filmed micro assemblies where operations of general load-unload of operation positions are performed by robot-operator

Duration of operations for surface preparation, cleaning and regeneration of the discharged water and other technological liquids and solutions for such flexible automatic manufacturing module is even more critical. Taking into consideration proved significant advantages of aerodynamic foam generators in comparison to other devices with same applications, in many of which the foam is a product of chemical reagents, one may suggest that usage of aerodynamic foam generators where foam is formed without chemical reagents and thus this process is more stable and more preferable and efficient, specifically for this type of special technological equipment. As main requirements for the foam quality the properties of controllable threedimensional homogeneity, stability 6 and guaranteed speed of formation can be considered.

\section{Approach Methodology}




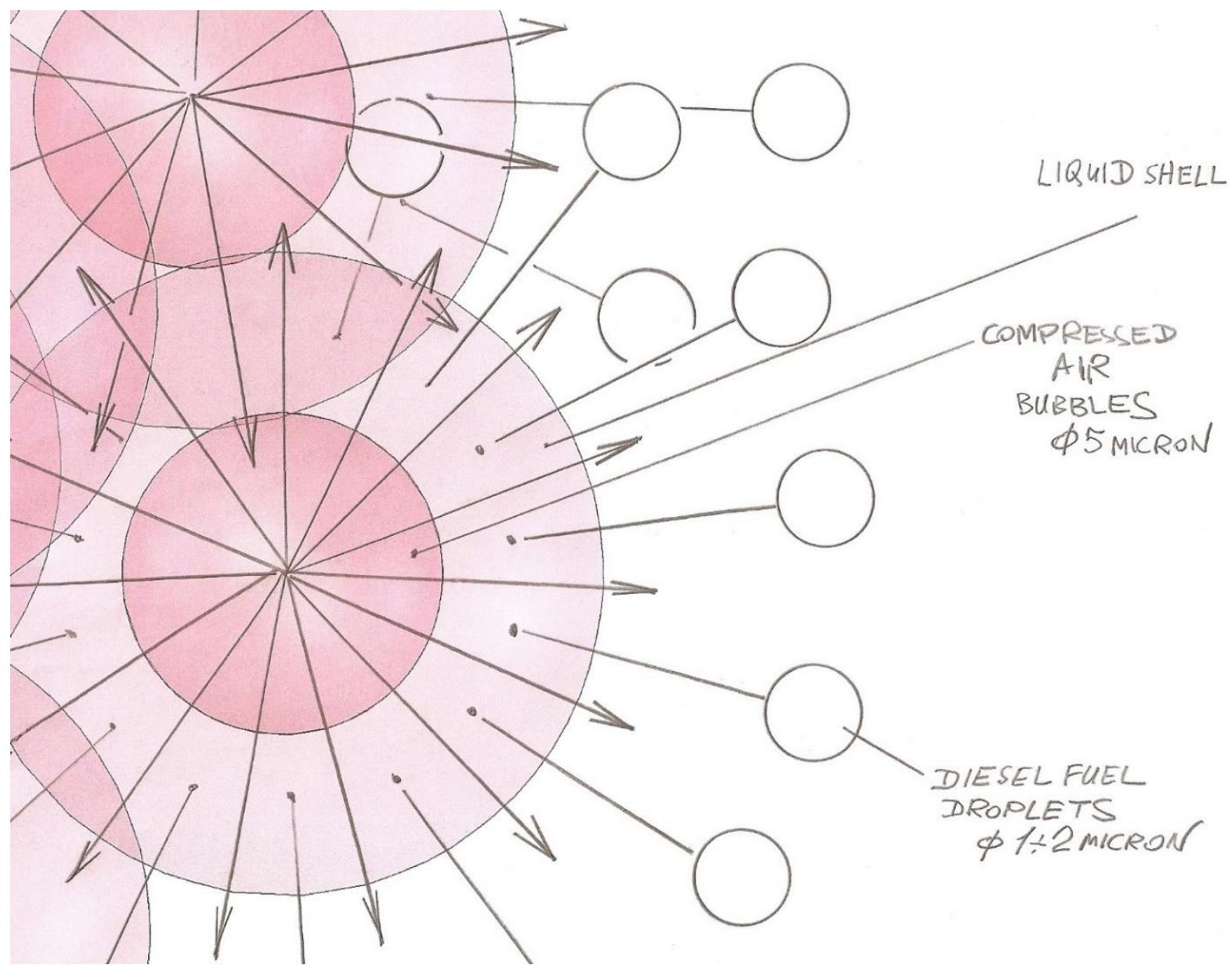

Figure 2. Principal diagram of active foam structure, obtained from aerodynamic foam generator during a process of diesel fuel activation before its injection into combustion chamber

Figure 2 shows that the foam obtained from aerodynamic foam generator initially consists of spherical capsules which have a core of compressed air with a diameter of 5 microns with a coating of diesel fuel which also has a thickness of 5 microns which after injection is disrupted by the streams of dilating air to the parts of size 1-2 microns.

Aforementioned statements require clarifications of the formulations.

For defining formulations for the aerodynamic foam generator:

Foam generator (device for aerodynamic foam generation) which includes:

- At least one container with liquid to be foamed

- At least one pump connected to the inner volume of specified container 
- Pipeline connected at the input with the output pump branch pipes and having built-in in its inner-volume at least two consecutive stages of the volume effect on the turbulence level of the specified stream and inserted in object consuming the foam (objectconsumer)

- Located in the direction of the liquid stream in the pipeline hydro-dynamic turbulence amplifier-mixer

- Located after the hydro-dynamic amplifier of the turbulence level in the direction of the liquid stream in the pipeline connected to the source of compressed air, aerodynamic stimulator of three-dimensional micro-turbulence in the stream of specified liquid

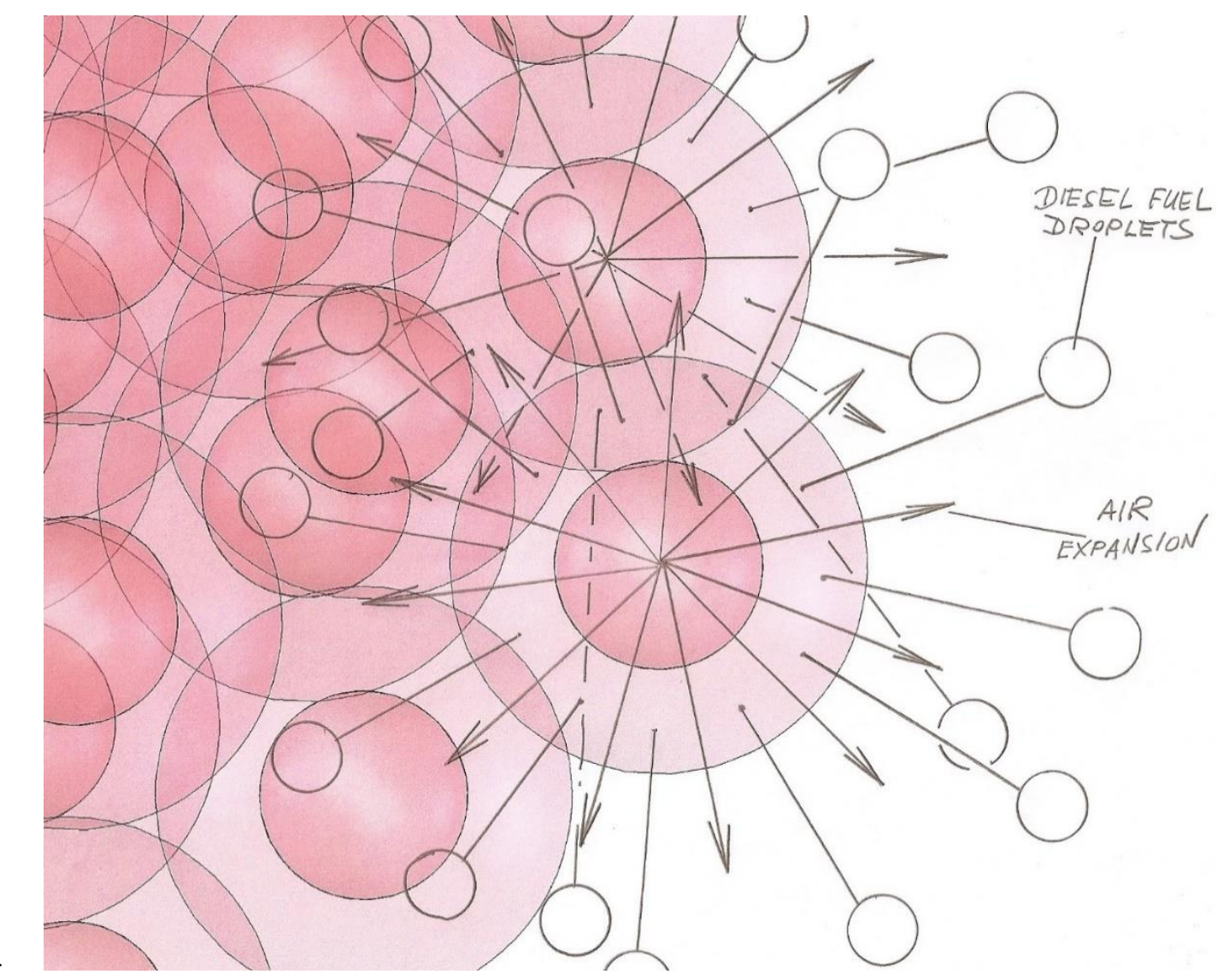

Figure 3. Detailed principal diagram of the active foam structure of the foamed diesel fuel with specification of the versions of the three-dimensional compressed air expansion from foam capsules after injecting in the thermo-dynamic object combustion chamber 


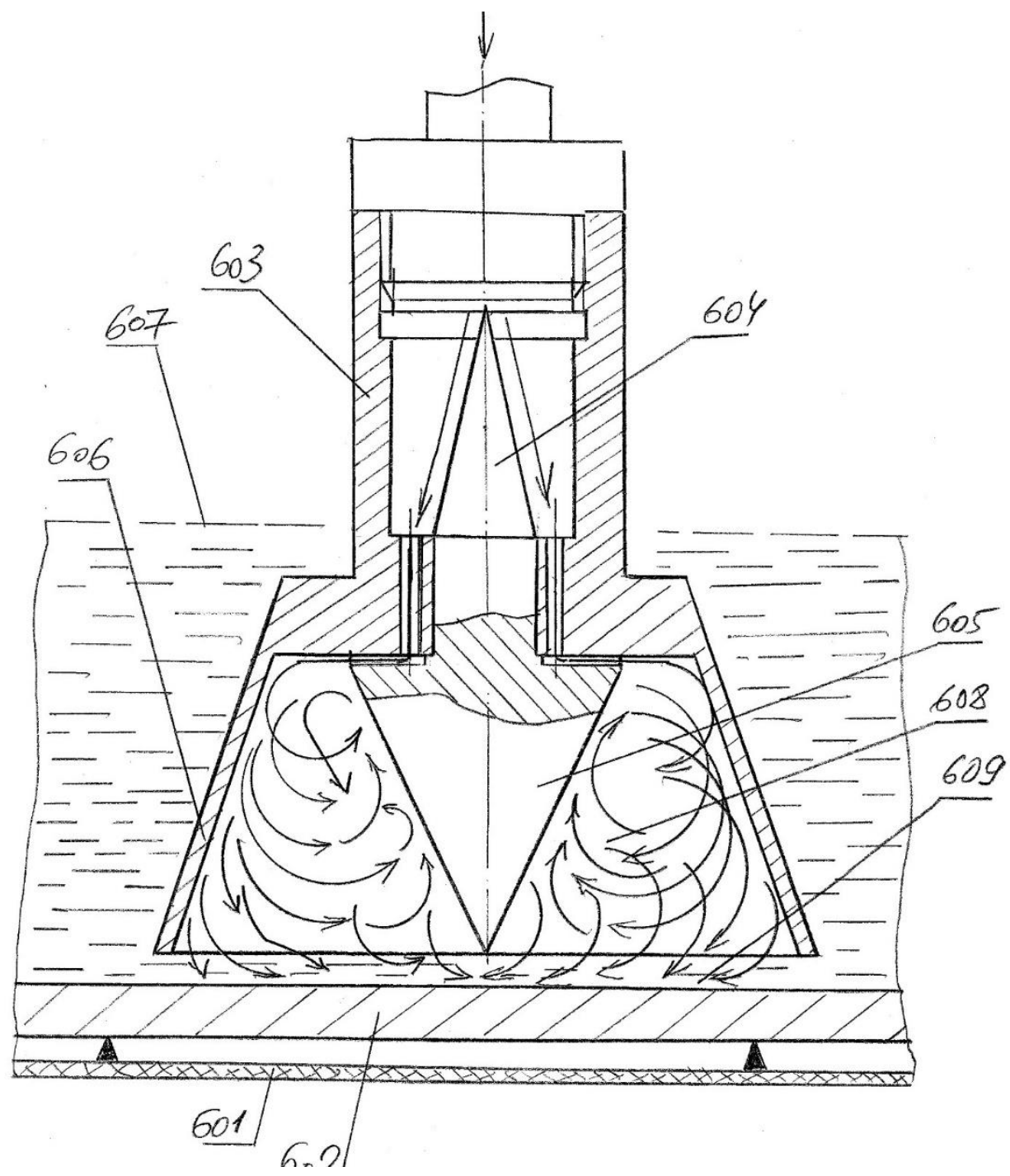

Figure 4. Model of a one type of aerodynamic foam generator which is used as an activating and cleaning device in the equipment for surface preparation without using of chemical reagents

Numbers in the Figure 4 stand for:

- 601, - container base

- 602, - cleaning surface

- 603, - aerodynamic foam generator body

- 604, - reflector that is located first in the direction of compressed air flow, which uniformly dispenses the compressed air stream over the whole body diameter of element 603 
- 605, - reflector forming the annular zone for foam formation in the toroidal space constrained by the conical surface of reflector 604 and inner conical surface of the funnel 606

- 606, - conical funnel of the aerodynamic foam generator body which constrains the toroidal space where foam is forming

- 607, - technological liquid, where under the affect of the aerodynamic foam generator operation the toroidal three-dimensional vortical volume of dynamic foam is formed which interacts with all the surfaces participating in the process and has an effect of hydro-dynamic cutter which in terms of properties and effects is equivalent to the properties and effects of a vortical pipe formed geometrically by conical surfaces of 605 and 606

- 608, - Inner volume where happens the formation of the aerodynamic foam with dynamic properties of vortical pipe and equivalent to it hydro-dynamic cutter, distribution of which is constrained and caused by specified toroidal space

- 609, - transition hydro-dynamic zone between toroidal space where the aerodynamic foam is forming and surface of detail 602 , which is a subject to processing As the invention of aerodynamic foam generator has a structure of apparatus, program, system and the associated application method, the part of invention formula is provided, which is related to associated method of the liquid agent foaming in various compositions and modifications:

The way of liquid agent foaming consisting of at least one liquid component primarily of an organic nature containing the insertion of specified liquid agent in the pipeline and formation of the dynamic stream of the specified liquid agent in the pipeline, insertion of the stream into the input cone of the hydro-dynamic turbulence amplifier-mixer, stream acceleration in the capillaceous channels and its reaching of the developed turbulence state with simultaneous injection of the three-dimensional stream micro-turbulence of the compressed air in the aerodynamic stimulator, compressed air stream acceleration in the capillaceous channels uniformly distributed over the cross-section area of the specified pipeline, formation in the compressed air acceleration zone of the area of the reduced pressure and suction in the created local zone of rarefication of all the volume of formed turbulence stream and micro-bubbles 
formation in the stream at the output of a zone with reduced pressure, stream saturation by the gas micro-bubbles and filling the whole pipeline volume with the foamed liquid agent.

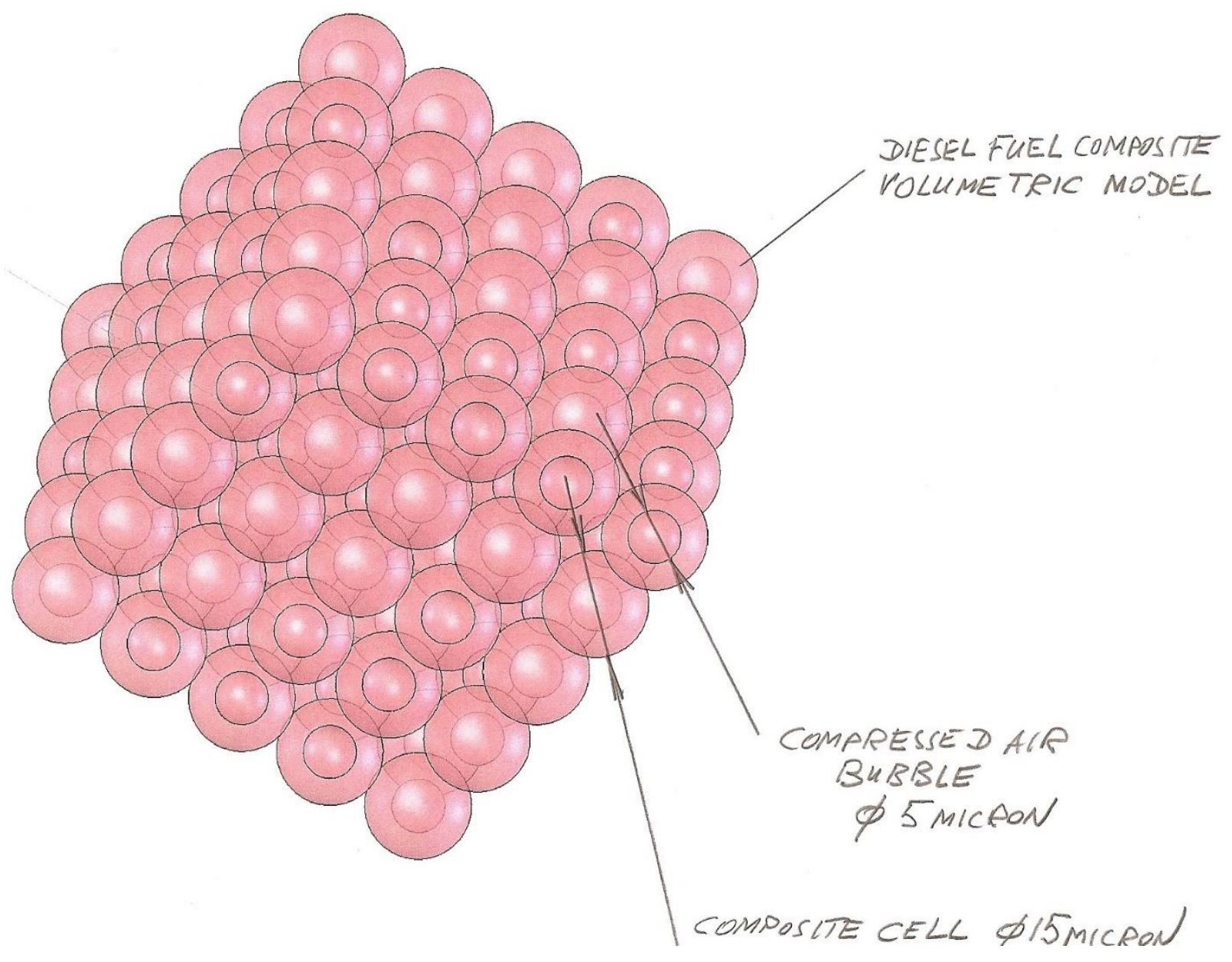

Figure 5. Three-Dimensional foam model obtained via aerodynamic foam generator using the aforementioned method for liquid agent foaming 


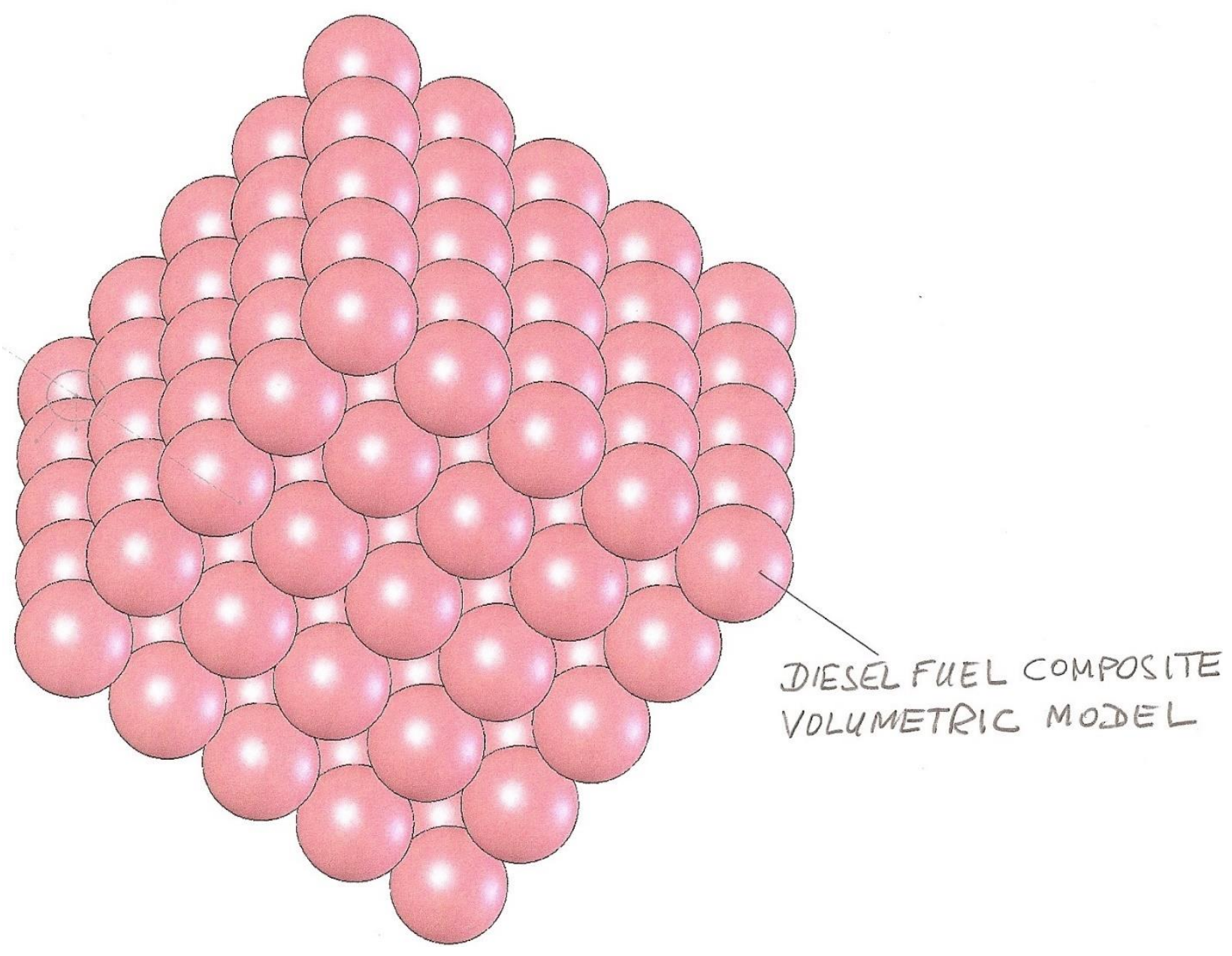

Figure 6. Three-dimensional foam model of a diesel fuel and air.

In the figure 6 the size of foam capsules that consist of core (which is a bubble of compressed air with diameter of 10-25 micron) and its spherical cover with a thickness of 10 micron leading to the total capsule diameter of 30-45 micron. Such structure gives this foamed fuel composite significant qualitative and operational advantages. It also allows to achieve the significant fuel economy with reduced toxicity of the exhaust gases when using the aforementioned fuel composite.

Foam generated with this method consists of many spherical capsules (composite elements) each of them consists of at least two components. Inside each capsule there is a bubble of compressed air under the pressure of 3-7 bar with a diameter of 5 microns. As the membrane of foamed liquid of liquids mixture has a thickness of 5 microns on average, the overall capsule size is 15 microns. This capsuled micro-foam by all its properties can be considered a composite 
and aerodynamic foam can also be considered a composite material with properties of compressible liquid.

Coming back to the topic of analytical analysis of novel foam properties obtained via aerodynamic foam generators, we can conclude that the obtained structure due to micron size of the spherical foam capsules can be classified as a compressible liquid.

If this foam is used as a fuel, then such properties allows it to solve one of the most difficult problems of injection fuel supply stability leading to reduction of vibration amplitude and fuel mixture pulsation in the fuel pipeline. This helps to increase the resulting net power efficiency, reduce fuel consumption and improve level of ecological safety of the combustion process while reducing the level of aerodynamic noise.

It is also important to analyze the required injection pressure. Experiments indicated that the required injection pressure in a range of 1600-2000 bar which is used for injection in the serial diesel engines when using a fuel composite as a composite fuel foam can be reduced to 9301000 bar while maintaining analogous output parameters of the combustion process. Such pressure reduction allows to reduce fuel consumption by 8-10 percent and also allows to simplify the structure of the sealings of combustion chamber or diesel engine cylinders.

These factors certainly need further experiments with additional usage of more complex combinations of liquid fuel, for example using the mixture of diesel fuel №2 with methanol or ethanol, and also with different combinations of mixture and biofuel. Mixtures and foam formation with more heavy fuel types as well as with varieties of masut (fuel oil) are the targets of further steps of research and experiments. 


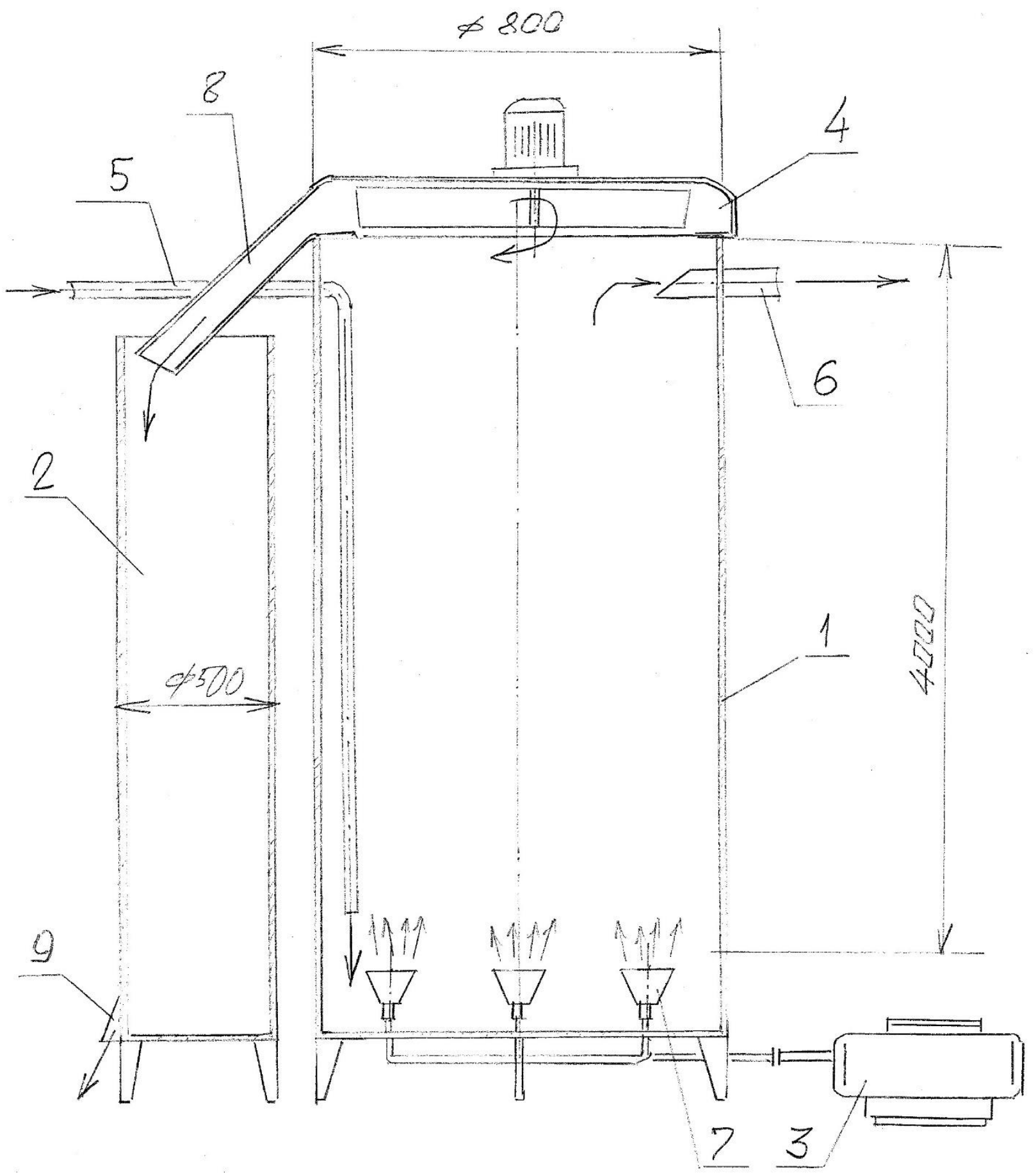

Figure 7. Principal diagram of the device for using the aerodynamic foam generator for various manufacturing processes including regeneration, cleaning and flotation processes and so on. 
Signs of significant novelty discovered during the comparative analysis of the suggested technical object and existing technical objects identified during patent search:

- foam generator is located inside the inner volume of the pipeline which is intended for supplying liquid for foaming or for supplying components for mixing with subsequent foaming;

- The process of mixing and subsequent foaming is based on the physics principle of local pressure reduction in the zones of active effect on the dynamic flow of components of liquid for mixing and subsequent foaming or for increasing turbulence level and subsequent aerodynamic foaming;

- for increasing turbulence level and increasing the value of Reynolds number method of stream division into the set of identical micro-streams was used. These micro-streams are uniformly distributed over the pipeline cross-section, and their linear speed is by the order of magnitude higher than the speed before division;

- method of staged consecutive operations for increasing the turbulence level in the stream and then for creating zones with reduced pressure sequentially ordered in the direction of stream flow was used. These zones gulf the stream with high intensity, sharply increase its speed and/or mix or foam the liquid or mix of liquids moving in the stream;

- all structural properties and connections for hydro-dynamic turbulence level amplifier and for aerodynamic foam generator during analysis of its discovered analogues indicated its complete novelty. Furthermore, the principle of the counterflow supply of liquids or liquid and compressed gas can be considered a pioneer as it was not found in any of the analogues.

\section{Invention Analysis by Methods of Theory of Inventive Problem Solving (TIPS) and Algorithms of Inventive Problem Solving (AIPS)}

1. Fundamental distinctive properties of the developed technology

1.1. Both liquid and gas components are used simultaneously as a working body;

1.2. Local rarefaction zone is created on the dynamic border between gas and liquid;

1.3. Mechanical two-sided reflector performs several functions for both gas and liquid: separation, join and formation;

1.4. In local rarefaction zone the dynamic contact of two mediums happen. It results in kinetic diffusion of the gas medium into liquid one due to higher dynamic characteristics of the gas medium stream; 
1.5. Kinetic diffusion of a gas medium into the liquid medium happens with detaching of gas bubbles from the stream and their free migration in the liquid medium volume following vortical trajectories;

1.6. Trajectory pattern of the air bubbles is formed by the set of symmetrical reflectors that are concentric with the dynamic border between two working mediums;

1.7. Dynamic border between two mediums has a shape of closed annular area. Its elements' geometric coordinates lie in the three-dimensional system which is concentric and coaxial to the channel that supply the gas working agent.

2. Goals which this invention accomplishes.

2.1. Efficient formation of plenty of microscopic air bubbles in the volume of liquid working body. These bubbles are uniformly distributed over the volume of liquid working body and have high kinetic energy.

2.2. Efficient formation of the specified bubbles while having minimal energy consumption and usage of the physics principles amplifying this effect.

2.3. Obtaining the optimal results while having the minimal dimensions of the operating devices.

3. Ways of obtaining same effect using other technical devices.

3.1. All seven principal distinctive properties together fully ensure achieving the specified goals;

3.2. In order to theoretically achieve same results using other technical devices that are not equivalent to the suggested device it is necessary to completely change patterns of all seven distinctive characteristics. Furthermore, the nature of the structural and technological interconnections between those characteristics must be changed as well, which is practically impossible to achieve.

4. Changing elements in the suggested technology components in order to obtain a patent.

4.1. The invention represents tight and technically sufficient interconnections between seven principal distinctive traits;

4.2. Any principal change should change the nature of their interconnections which eliminates obtaining the specified effect and the operability of the device itself can be questioned; 
4.3. Any change in the device dimensions does not change the general operation principles of the device and corresponding technology. Thus, it cannot ensure the device operability which is comparable to the suggested invention.

5. Necessity of using dimensional and numerical dependencies in the invented device design instead of general definitions and distinctive properties and ways it can make a patent stronger.

5.1. Transition to the specific dimensional characteristics from the principal and generalized characteristics significantly weaken the invention;

5.2. In order to introduce any dimensional changes or constrains in addition to the suggested principal differences, it is necessary to significantly weaken the protective functions of the patent and constrain the flexibility of its application.

6. The principle and the related Bernoulli effect are only the consequence of applying all seven principal distinctive characteristics and their structural and technological interconnections.

7. All empirical formulae and dimensional dependencies and combinations are shown in the figures of the corresponding publications and can be considered only in scope of principal distinctive properties and they do not bring any value on their own.

\section{Conclusion}

After analysis by methods of Theory of Inventive Problem Solving (TIPS) and Algorithms of Inventive Problem Solving (AIPS) the suggested technology can be considered as a pioneer in terms of technological complex solution (foam generation method); nature of foam properties and characteristics (product characteristics); device properties and characteristics (foam generator); process of foam usage in the formation of thermo-dynamic equipment fuel system. Capabilities of formation of the qualitative foam aspect and almost instant high-quality foam formation open new perspectives in the development of new layout arrangements in special technological equipment especially in the conditions when it is necessary to create an infrastructure that corresponds to utilization of artificial intelligence and neural networks in combination with modern processor and programmable controller devices in technical systems. 


\section{References}

1. David Scott Alburty et al., "Portable Concentrator." U.S. Patent 20110197685, issued August 18, 2011

2. Robert P. Munroe et al., "Foaming Cream Emulsions." U.S. Patent 20190209473, issued July 11, 2019

3. Yousuke Okada et al., "In-Situ Foaming System for Forming Flame-Retardant Polyurethane Foam in Situ.” U.S. Patent 20190194413, issued June 27, 2019

4. Ryohei Aoyama et al., "Foaming Dispencer." U.S. Patent 20190191936, issued June 27, 2019

5. Frank Prissok et al., "Microwave Foaming." U.S. Patent 20190177501, issued June 13, 2019

6. Paul Heffernan et al., "Post-Foaming Mild Cleansing Composition." U.S. Patent 20190117527, issued April 25, 2019

7. Justin Stewart et al., "Systems for Mixing a Liquid and Related Methods." U.S. Patent 20190060855, issued February 28, 2019

8. Jianzhi Yan, "Foam Soap Dispenser." U.S. Patent 20190008331, issued January 10, 2019

9. Dirk Schmitz, "Apparatus and Method for Producing Compressed Air Foam for Fire Fighting and Fire Fighting Apparatus." U.S. Patent 20180207458, issued July 26, 2018

10. Norihiro Morikawa, "Foamed Resin Production Method and Production Equipment Thereof.” U.S. Patent 20180200927, issued July 19, 2018 